\title{
Probing the molecules of the mind
}

\author{
Barry Vincent Mendelow
}

Emeritus Professor, Faculty of Health Sciences, University of the Witwatersrand and National Health Laboratory Service, Johannesburg, South Africa

\begin{abstract}
The molecular dimension of biology has been finding practical applications in all fields of medicine over the last few decades, but arguably one of the most challenging and exciting areas is that of molecular psychiatry. Much progress has been gained from traditional linkage studies into gene candidates for various psychiatric disorders, including schizophrenia, bipolar states, bulimia, autism and attention deficit hyperactivity disorder. This review outlines basic principles of molecular medicine from the perspective of the human genome project and its role in enabling rapid progress in the understanding of molecular neuro-anatomy, physiology and pathology. These insights have facilitated rather than complicated the diagnostic process, and have paved the way, in what has been termed the "post-genome era", for a rational approach to therapy in a growing range of disorders. A patient's inherited genetic constitution is but one aspect of molecular medicine, and rich insights are also being gained into the operation of the genome as it interacts with its environment, including its pharmacological environment. From parallel processing technologies and genomic expression profiling, massive knowledge is being gained into the anatomical and physiological patterns of gene expression in different tissues and organs, their derangements in disease and their pharmacological manipulation. The brain is no exception, and an appreciation is being accumulated of the three dimensional anatomical localization of cerebral gene expression patterns, of great relevance to a fundamental understanding of neurobiology. From this new paradigm and others, insights are now being gained into the molecular biology of a variety of topics previously thought to be beyond the scope of molecular dissection, such as language and emotion.
\end{abstract}

Keywords: Gene expression, Micro array, Pharmacogenetics, Psychiatric

Received: 10.01 .05

Accepted: 14.02 .05

\section{Introduction}

The publication of the sequence of the human genome, in February of 2001 was a milestone in the history of science, whose significance in history has been compared with landing humans on the moon. Just as with the first lunar landing, the event itself held a special symbolic significance for humanity, but also marked the beginning of a wave of practical spin-offs for ordinary society. Direct benefits of the human genome project for human health and medical practice are only now gaining momentum. Some of these have been reviewed from the perspective of the neurosciences. ${ }^{1}$ The purpose of this review is to sketch some of the basic molecular and cellular concepts underpinning the insights that are now being gained, in what has been termed the post genome era of functional genomics, into the molecular machinery of the mind. For a full account of the basics underlying molecular medicine and molecular biology, the reader is referred to recent texts for an overview ${ }^{2}$ or a detailed treatise. ${ }^{3}$ This review begins with a brief synopsis of what has been called the central dogma of molecular biology from a gen-

\section{Correspondence:}

Prof BV Mendelow, Faculty of Health Sciences, University of the

Witwatersrand, 7 York Road, Parktown, Johannesburg, South Africa.

email: mendelowbv@pathology.wits.ac.za eral cellular perspective, using the analogy of information technology systems to illustrate basic molecular principles and mechanisms of intra- and intercellular communications, proceeds to a molecular classification of general pathology and concludes with a discussion of some of the exciting new tools which are unravelling genomic functions in the nervous system, with special reference to clinical psychiatry.

\section{Basic cell and molecular biology}

Arguably the most important conceptual foundation of modern biology is that genetic information is essentially digital in nature. As it happens, the Information Technology (IT) organizational paradigm, built as it is on the special semiconductor properties of silicon, is a convenient framework for a modern understanding of cell and molecular biology and biotechnology, based as they are on the special properties of carbon, and vice versa. The analogy of computer viruses, which traces its origins to the 1970's ${ }^{4}$ introduced a useful model both for the understanding of information technology in the language of biology $y^{5}$ and of molecular biology in the language of information technology.

Multicellular organisms are each clones of a single cell, whose genome is effectively the digitally encoded design blueprint for the whole organism. The genome of any organism, be it a microorganism, plant or animal, is the sequence of the fundamental digital units of information that constitute the specification for the construction and function of that organism. 
Silicon-based computer information is based on a binary code, in which each fundamental unit of information can exist in one of two states, on or off. These are termed binary digits or bits. With carbon-based biological information systems, the fundamental units of information are also digital, but instead of being based on a binary code, they are based on a quaternary (base four) system. That is, each position of the block of code is occupied by one of four different possible quaternary digits (qits). These are the chemical bases adenine, guanine, cytosine and thymine (AGCT), which when linked together by deoxyribose sugar units via phosphate groups into a long string, constitute DNA. The understanding of the molecular anatomy of DNA that was provided by Franklin, Wilkins, Watson and Crick paved the way for the understanding of its functions. Thus, information encoded in the sequence of the four bases is copied according to the base-pair principle, viz A forms hydrogen bonds specifically with $\mathrm{T}$ and vice versa, while $\mathrm{G}$ forms hydrogen bonds specifically with $\mathrm{C}$ and vice versa. In this way each strand of DNA is strongly bound through specific hydrogen bonds to its complementary strand of the double helix. The human genome comprises approximately 3 billion such base pairs, each human differing from each other human by a fraction of a percent of these (except in the case of identical twins, whose primary sequences are identical to each other). It is these differences that encode the phenotypic differences between us.

Human DNA is packaged into 23 pairs of chromosomes, each of which is analogous to archival data storage devices such as the magnetic or optical disc. DNA, like such media, is chemically and physically stable and thus is well suited as an archival form of data storage device. On its own, DNA is no more capable of fulfilling any action than the software of an optical or magnetic disc. Action requires that one functional stretch of the information, a computer program (analogous to a gene), must be read from the archive into a working copy. The human genome archive contains about 30,000 genes or programs. Each cell uses only a small fraction of the complete archive at any one time, the precise subset of genes in use defining the lineage of the cell and its functional activities at that time. Thus, a red cell precursor, for instance, would need to use the genes for globin, erythropoietin receptor, G6PD and any other proteins specifically needed by the red cell in order to perform its unique actions. A nerve cell uses a different subset, including the genes for the various protein components of the synapse, which it draws from the archive.

In a computer, the clicking of the mouse frequently initiates the process of loading a program from the archival storage on the hard drive into the working storage space provided by RAM, or Random Access Memory. The equivalent process in biological systems is that of gene transcription, which is frequently initiated by the engagement of a signalling molecule by a cellular receptor. The process of loading a program from a magnetic or optical disc into Random Access Memory (RAM) is thus analogous to the process of transcribing a gene, whereby the relevant stretch of information is copied from DNA into RNA. RNA, like RAM is an unstable storage medium, ideally suited to be used briefly and discarded. The RNA strand is similar to that of DNA except that each nucleotide sugar contains an extra oxygen atom, which effectively destabilizes the molecule, such that RNA is rapidly and readily degraded. The other significant difference concerns the substitution in RNA of uracil for thymidine.

Although possessed of certain catalytic functions of its own in the case of ribozymes (RNA based enzymes), the best understood function of RNA is as an intermediate interpreter between the information stored in DNA and the amino acid sequence of proteins. A cytoplasmic device, the ribosome, with functional properties that are analogous to a central processing unit or CPU (which translates information in one form into another), accomplishes the translation of the nucleic acid sequence of nucleotides into the protein sequence of amino acids. A triplet codon of three nucleotide bases in a row is used to designate each of the 20 amino acids available, just as an octal code of eight binary digits in a row (a byte) is used to designate each of the 26 letters of the alphabet in the ASCII code (American Standard Code for Information Interchange) used universally to represent text. In both biological and IT systems, the number of secondary data states $\left(4^{3}=64\right.$ for biological systems, $2^{8}=256$ for IT) is sufficient to encode the relevant amino acid/letter of the alphabet and leaves plenty of space for punctuation (eg stop codon/full stop) and redundancy (Table 1).

Each protein, whether for the cell's internal use either as a structural component, a receptor or as an enzyme, or for export, such as immunoglobulin, is ultimately an encoded product of the gene in the DNA sequence. Some of the internal or membrane associated proteins constitute receptors for molecules exported from other cells. The precise set expressed is a function of the lineage of the cell in question. In this way, the receptor apparatus of each cell is more akin to a keyboard than a mouse, because the receptors constitute a receptor array, and the behaviour of each cell is orchestrated by which specific set of keys is "clicked" and in which specific order.

Table 1: Comparison of Silicon versus Carbon based information and communication systems

\begin{tabular}{|l|l|}
\hline \multicolumn{2}{|c|}{ Information Technologies } \\
\hline Silicon & Carbon \\
\hline Bit (On/Off) & Qit (ACGT) \\
Byte (8 Bits $=2^{8}=256$ states) & Codon (3 Bases $=4^{3}=64$ States) \\
Letter/numeral & Amino acid \\
Program & Gene \\
Load & Transcribe \\
Magnetic/optical(CD/DVD) disc & DNA \\
RAM & RNA \\
Run & Translate \\
Data output & Protein \\
Mouse & Receptor \\
Keyboard & Receptor array \\
Data & Coding sequence \\
Instructional set & Promoters and enhancers \\
CPU & Ribosome \\
Bug & Mutation \\
Virus & Virus \\
\hline & Communication \\
\hline Bandline (Telkom) & Technologies \\
Cellular Communication (GSM) & Neural \\
\hline & Endocrine \\
\hline
\end{tabular}




\section{Cellular communications}

For the assemblage of trillions of cells that constitute a person, there needs to be an effective system of communications for the organism to function as a cohesive unit. Once again the biological system has close analogues in the world of Information and Communication Technology (ICT). Three main intercellular communication systems are represented, these being the neural system, which is functionally equivalent to the Telkom landline telecommunication system, the endocrine system, which is analogous to the wireless global system of mobile (GSM) communications, and the paracrine system of short range intercellular communication, which acts in the same sort of fashion as the $\mathrm{Wi}-\mathrm{Fi}$ wireless local area networks (WLAN), or the Bluetooth personal area network (PAN). The paracrine system is of special relevance in the regulation of the immune system and in stem cell microenvironmental niches, such as the bone marrow, where cells communicate at very short range. The biological telecommunication exchange is represented by the hypothalamic/pituitary axis.

\section{The genome and the environment in health and disease.}

The effect of the genome on the structure and function of the organism which it encodes is of such a profound nature that modern pathology is conveniently classified into two broad groups of disorders, these being genomic and non-genomic (Table 2). It is increasingly apparent that many diseases that are not inherited are nevertheless "genetic" in the sense that genes play a major role in their cause, course, diagnosis and treatment. Most if not all diseases not encompassed in this simple scheme prove to be combinations of the basic elements.

\begin{tabular}{|l|l|}
\hline \multicolumn{2}{|l|}{ Table 2: Molecular Classification of Disease } \\
\hline Genomic & Non-genomic \\
\hline $\begin{array}{r}\text { Inherited mutations } \\
\text { Acquired mutations } \\
\text { Neoplastic } \\
\text { Degenerative }\end{array}$ & Trauma \\
Infections & Toxins \\
& Nutrition \\
\hline \multicolumn{2}{|c|}{ Combinations } \\
\hline
\end{tabular}

\section{Genomic disorders}

The understanding that inherited diseases are genetic in nature is self-evident and needs very little elaboration. Thus, any inherited digital "bug" (mutation in the DNA), such as the single base substitution of the sickle cell anaemia gene, (adenine to thymine in the gene for beta globin) is copied faithfully to the entire clone of cells constituting the patient. Only those cells that transcribe globin genes, ie red cell precursors, will ever transcribe this gene, but when they do, the mutation is translated, via mutant RNA into mutant beta globin protein, which has a water-insoluble valine at the 6th position of its amino acid sequence instead of the normal water-soluble glutamic acid. The globin protein so produced has compromised water solubility and precipitates into long insoluble needle-like molecules, which distort the red cell into the characteristic sickle shape. Similar logic applies to the pathogenesis of all inherited genetic disorders, whether single gene diseases such cystic fibrosis or haemophilia, or polygenic disorders such as most common inherited predispositions to heart dis- ease or stroke.

What is not quite so self evident, however, is that genes also play a central role in many other disorders for which there are no heritable factors. Although inherited defects in DNA repair enzymes predispose to malignancies in some families, most cases of cancer, are not inherited, yet the role of genes (oncogenes) is pivotal to their nature. In every case of cancer that occurs, or indeed any neoplastic tumour, benign or malignant, the fundamental lesion boils down to the mutation of one or (usually) more than one acquired DNA mutation in a single cell, whether or not there is any inherited defect in the cell's DNA repair machinery. The nature of the mutation is such that the mutant protein it encodes effectively frees the cell from the normal constraints that are imposed on the survival and or growth of individual cells in the multicellular context. Typically the mutant protein is one that is involved at one or another level in the transduction of growth signals from the exterior, via growth factor receptors through to cytoplasmic enzymes and nuclear transcription factors to the nucleus of the cell to initiate cell division and or survival. The mutant cell is permitted to survive or proliferate by cell division, each division copying not just the cell but along with it the mutant gene that initiated its inappropriate division in the first place. The daughter cells are thereby similarly endowed with the same capacity to survive and or grow independently of the actual needs of the body of which they form part. In this way, by successive divisions, a clone is generated, each sharing the identical genetic mutation and behaviour tendency. It is this clonal mutation that lends itself to the rapidly evolving technology aimed at the detection of such mutations in the DNA to establish the diagnosis of any particular type of cancer and to tailor its therapy to the specific patient. ${ }^{6}$ Recently these insights have been translated into therapeutic benefit by the systematic and rational design of new drugs targeting molecular disease pathways. ${ }^{7}$

Other acquired mutations that result in genomic diseases include many examples of degenerative disorder, and even the aging process itself appears to be largely a consequence of the cumulative acquisition of DNA mutations. A particularly interesting example of how this works is seen in the progressive shortening of chromosomal ends, or telomeres, with successive cell divisions, which effectively imposes a limit on the number of cell divisions available to a cell line before cellular senescence and death occur. ${ }^{8}$

Other major disease groups that are fundamentally genomic in nature include the entire spectrum of infectious disease, in which specific infectious micro-organisms exploit molecular strategies to cause disease in human hosts. The molecular dimension is fundamental in understanding such diseases, in establishing a specific diagnosis by demonstrating the presence of organism-specific DNA or RNA sequences in samples from patients, and in identifying the nucleic acid mutations that confer particular patterns of drug sensitivity or resistance.

\section{Non genomic disorders}

Conceptually these are simple to understand, and although molecular technology is generally not central to the pathology or diagnosis of this group, most have a molecular dimension. For instance, wound healing following trauma is orchestrated by the transcription of many genes intimately involved in inflammation, angiogenesis etc, while the metabolism of many drugs and toxins is very significantly in- 
fluenced by inherited genetic factors. Undoubtedly this underlies a number of instances of drug dependency with inherited predisposition.

\section{Combinations}

This group includes disorders such as autoimmune disease, which may constitute a combination of inherited and infectious genomic elements, complicated, at least in some cases, by clonal expansion (? neoplastic transformation) without overt tumour formation in cells of the immune system. In other abnormal states, genomic and non-genomic factors interact. In this way the molecular blueprint specifying each unique human, animal plant or microorganism, is "choreographed by the context" of its experience and environment. ${ }^{9}$ This may have special relevance to the integration of molecular psychiatry into traditional paradigms.

\section{Basic molecular tools}

While it is not the intention of this review to delve in detail into technological issues, there are nevertheless a few methodological concepts that require mention and very brief description. These methods have been applied across the board in medical diagnostics, and their application in the identification and understanding of psychiatric disorder is a particularly fruitful arena of current and future research.

One of the most useful tools in the arsenal of the molecular biologist is that of the restriction enzymes. These are bacteria-derived enzymes with the property of cutting nucleic acids at identifiable sites by recognizing specific palindromic sequences (viz those reading the same in reverse direction on the complementary strand), thus effectively chopping DNA into manageable sizes for further analysis. There are a number of additional tools, including gene cloning, library construction, Southern blotting (of DNA), Northern blotting (of RNA) and Western blotting (of proteins) and subsequent probing methods to define the presence of a particular predefined sequence in these blots. These methods are beyond the scope of this review and for an account of their scientific basis, the reader is referred elsewhere. ${ }^{10}$

The Nobel prizewinning technique of Polymerase Chain Reaction (PCR) has revolutionized the field of medical diagnostics, because it permits the rapid, accurate and exquisitely sensitive detection of specific nucleic acid sequences that are definitive of any genomic disease. This applies whether the disorder is inherited, neoplastic, infectious or combination of genomic factors. The basic principle is the amplification of a defined sequence by iterative cycles of duplication, leading to exponential growth in the number of copies of the sequence in question. It is convenient to visualize PCR as a kind of culture system, where instead of growing microorganisms, specific, predefined nucleic acid sequences are "grown". The sequence could be that defining HIV, or drug resistant HIV, or haemophilia, or the specific mutation defining chronic myelocytic leukaemia for instance. It can also be used in forensic application because the sequence is an unambiguous, person-defining property of even faint traces of DNA found in any material such as tissue, blood or other body fluid or its residue, DNA being remarkable stable under a range of environmental conditions. The great beauty of the technique is that no matter what the disease, the method for its detection is fundamentally the same.
The specifics are regulated by defining the starting point of amplification by including within the reaction mixture small primer sequences, without which amplification (by insertion of new nucleotides in the complementary sequence of the template) does not occur. If the target sequence is present in the test sample, amplification will occur - if it is not present, amplification doesn't get started. By repeating the process twenty times, for instance, about a million copies $\left(2^{20}\right)$ of the sequence are generated. The identification, by a number of techniques, of this greatly amplified number of molecules with the defined sequence is thus greatly facilitated. The result can be simply qualitative (is HIV present in the sample or not?) or quantitative (how many copies of the viral sequence were present in the test sample?)

With regard to psychiatric disorders, PCR and other methods for the detection of specific sequences have been very useful in establishing linkage between the presence of a specific sequence (either a marker sequence or a specifically mutated gene or polymorphic allele) in DNA and the presence of the clinical disorder in affected family members. The identification of the DNA sequences in this instance does not necessitate sampling of cerebral tissues and may be obtained from a blood sample. Specific genes may operate individually, or more typically as interacting sets. Examples of such linkages include schizophrenia ${ }^{11}$, depression ${ }^{12}$, Attention Deficit and Hyperactivity disorder ${ }^{13}$, bulimia ${ }^{14}$ and many instances of drug addiction. ${ }^{15}$

\section{Functional genomics in the post genome era}

One of the most powerful of the new technologies to emerge in recent times, again with applications extending throughout medicine but particularly applicable to probing the workings of the human mind, is that of gene expression micro arrays. Early critics of the projected impact of the human genome project on medical practice were quick to point out that the project had merely produced a catalogue of genes and that this informatiojn of itself held no tangible benefits for practising medical practitioners or their patients, just as a catalogue of car parts of itself is of little use to the motor mechanic. While this somewhat nihilistic viewpoint may be debatable, there can be no doubt that knowledge of which genes are being used in which cells at which times and under what circumstances is central to the understanding of many, if not all diseases. In other words, does the catalogue pave the way for the development of a comprehensive workshop manual which defines precisely how the various components operate together under normal and abnormal circumstances?

In the exploration of the mechanisms underlying the expression of genes under varying circumstances, what previously would have taken years to accomplish with respect to a single gene, can now be accomplished for the entire repertoire of 30000 genes within an afternoon, using the techniques of gene expression micro arrays. The technique is based on the positioning of a subset of genes or indeed the entire genome set, in a two dimensional array on a microchip. If messenger RNA from a cell or tissue is extracted, labelled and hybridised with this array, the specific spots, each representing a gene, to which this labelled RNA attaches (because of complementary base pairing,) are an indication of exactly which genes that cell or tissue is transcribing under those circumstances. By comparing different cell types, for ex- 
ample, one has an indication of the molecular differences between those cell types. The gene subset expressed by erythropoietic cells, for instance, is different from that expressed by neurones. Indeed that is precisely why cell lineages are what they are - because of the gene subset they express, all cells retaining a complete set of the whole genome but only using what their lineage prescribes. The number of data points under analysis can be enormous, especially in expression profiles of the entire genome, and the human eye is not capable of performing more than a rudimentary appraisal. For this reason, the arrays are scanned by electro-optical devices and the gene expression levels analysed by special software capable of discerning patterns and classes of expression automatically. From this parallel processing approach has emerged a new wave of hypothesis independent research and automatic class discovery, whereby crucial answers can be obtained to questions that haven't yet been asked!

By applying such techniques diagnostically it becomes possible to obtain a complete profile of exactly which gene subset is driving a particular tumour for example. Some of these genes which define the lineage of the tumour, which is one of the diagnostic tasks of the histopathologist, while others will define the pattern of gene expression of growth or survival control genes, which gets to the heart of understanding what makes that particular tumour tick. Such information is of immediate practical value in a diagnostic sense, but more importantly, however, it opens up a vast new vista of potential therapeutic strategies, all based on the goal of influencing the gene expression pattern in such a way as to restore normal cell behaviour.

By combining different parallel processing modalities researchers have begun to address gene expression patterns in different anatomical sites within the brain, normal and abnormal. ${ }^{16}$ Using autopsy material, they have produced gene expression brain scans, in which the expression patterns of any individual gene can be visualized throughout the brain, and abnormal patterns become immediately apparent. The method depends on the preparation of thousands of small cerebral blocks, termed voxels (volume elements), each of which is registered as to its exact anatomical location. By extracting RNA from these individual blocks and using gene expression micro arrays to evaluate the gene expression profile of each block, the data can be recombined into a genome expression scan of the entire brain, at a resolution which is set by the number of voxels prepared. This can number in the hundreds of thousands, with each voxel analysed for the quantitative level of expression of up to 30000 genes, in the normal state and in any defined clinical neurological or psychiatric disorder. The volume of data generated by this voxellation-expression technology is astronomical, but once again the burgeoning power of modern information technology is capable of sifting all these data and extracting meaningful conclusions. From this approach and also from a related approach of gene expression tomography, the concept of the neurome ${ }^{17}$ is evolving as a research database of information describing anatomically explicit cerebral function at a molecular level. For the elucidation of normal cerebral molecular physiology, this is exciting enough, but the prospect of using the same methodology to generate a new paradigm for the understanding of the molecular pathology of the mind is where the real impact on the practice of psychia- try will be found.

Using these methods, there is at last progress in the delineation of the molecular basis of schizophrenia for example. Complementing aspects of the linkage studies referred to above, gene expression patterns in schizophrenic brains are revealing a convergence on genes encoding protein components of the synapse, as the common molecular pathway for the development of schizophrenia. ${ }^{18,19}$ Thus, these workers propose that schizophrenia is the result of failure in the molecular assembly of a fully functional and appropriately adaptable synapse. Failure of any of the components of the synapse, whether because of inherited abnormality or acquired interference, or both, may have a similar clinical result, just as clinical bleeding may be the result of failure of any of the components of the coagulation cascade, whether because of inherited abnormality (eg haemophilia) or acquired interference (eg warfarin exposure). Insights of a similar conceptual nature have been gained using the same methodological approach of gene expression micro arrays in Alzheimer's disease $^{16}$ and bipolar disorder. ${ }^{20}$

Research of this nature is likely to generate currently unimaginable approaches to targeted pharmacological interventions in psychiatric disorders. Although the technique is currently restricted to autopsy material, it is not inconceivable that after appropriate ethical scrutiny in the light of costbenefit analysis, it could one day be combined with stereotactically guided, minimally invasive ultra fine needle sampling, and used in a diagnostic mode. It must be cautioned that that day has not yet dawned.

Gene expression micro arrays can be used to explore the mechanism of action of drugs. By extracting RNA from cells either exposed or not exposed to a drug, and applying the RNA to gene expression microarrays, knowledge is gained as to exactly which genes are influenced, positively and negatively, by the drug. The understanding of the mode of action of antidepressants ${ }^{21,22}$ and other medications of interest to the psychiatrist ${ }^{23}$ have similarly been revolutionised by these techniques.

Other insights relating to psychiatric drugs that the molecular revolution has spawned include the finding that different individuals inherit varying rates of general drug metabolism, such that standardized drug doses may be systematically inadequate in certain individuals and excessive in others. $^{24,25}$ This and other factors specific for the substance in question may also apply to inherited predisposition to drug dependency ${ }^{15}$, including alcoholism. ${ }^{26}$

In the further investigation of molecular neurophysiology, experimental situations have been set up to define the genetic mechanisms operative during sleep, in which state certain genes are actually up regulated, in contrast to the majority of genes amongst which differences were found where sleep was associated with diminished levels of expression. ${ }^{27}$ This implies that from the perspective of those genes that are up regulated, sleep is an active process! Other physiological processes whose underlying molecular machinery is being elucidated include the genomics of gender differences and sex drive $e^{28,29}$, general intelligence ${ }^{30}$ and memory. In this regard, it is of interest that an aspect of the process of forgetting appears to be active, with a molecule having been identified that actively induces the deletion of cognitive data. ${ }^{31}$ Even emotion appears to be genetically regulated, and 
relevant genes in this regard are being identified ${ }^{32,33}$, as are genes of importance in the use of verbal language. ${ }^{34}$

\section{The future}

Despite spectacular progress of late, it would be premature to state that there is now a comprehensive understanding of the molecular machinery of the mind. However, it is clear that a new dimension has been identified in the process of acquiring that understanding, and that is the molecular dimension. While there is still a long way to go, there are now extremely powerful tools available, whose application to the process of discovery was unimaginable a decade ago, and the rapidly expanding power of information technology is one of them. Moore's law stated that the power of information technology doubles every eighteen months, and the law appears to apply with equal validity to biotechnology as applied to molecular medicine and molecular psychiatry.

\section{References}

1. Cravchik A, Subramanian G, Broder S, Venter JC. Sequence analysis of the human genome: implications for the understanding of nervous system function and disease. Arch Neurol 2001; 58:17728.

2. Ross DW. Introduction to Molecular Medicine. 3rd ed. New York: Springer, 2002

3. Alberts B, Johnson A, Lewis J, Raff M, Roberts K, Walter P. Molecular Biology of the Cell. 4th ed. New York: Garland Publishing; 2002.

4. Gerrold D. When HARLIE Was One. New York: Ballantine Books, 1972.

5. Forbes, N. Imitation of Life: How biology is inspiring computing, MIT Press, 2004.

6. Carr KM, Rosenblatt K, Petricoin EF, Liotta LA. Genomic and proteomic approaches for studying human cancer: prospects for true patient-tailored therapy. Hum Genomics 2004; 1: 134-40.

7. Roskoski R Jr. STI-571: an anticancer protein-tyrosine kinase inhibitor.Biochem Biophys Res Commun2003; 309:709-17.

8. Brunori M, Luciano P, Gilson E, Geli V. The telomerase cycle: normal and pathological aspects. J Mol Med. Jan 4 [Epub ahead of print] 2005.

9. Mirnics K, Pevsner J. Progress in the use of microarray technology to study the neurobiology of disease. Nature Neuroscience 2004; 7:434-439.

10. Trent RJ. Molecular Medicine: an introductory test. 2nd ed. New York: Churchill Livingstone, 1997.

11. Owen MJ, Williams NM, O'Donovan MC. The molecular genetics of schizophrenia: new findings promise new insights. Mol Psychiatry 2004; 9:14-27.

12. Neves-Pereira M, Mundo E, Muglia P, King N, Macciardi F, Kennedy JL. The brain-derived neurotrophic factor gene confers susceptibility to bipolar disorder: Evidence from a family-based association study. Am J Hum Genet 2002; 71: 651-655.

13. McCracken JT, et al. Evidence for linkage of a tandem duplication polymorphism upstream of the dopamine D4 receptor gene (DRD4) with attention deficit hyperactivity disorder (ADHD). Mol Psychiatry 2000; 5: 531-536.

14. Nilsson M, Naessen S, Dahlman I, Hirschberg AL, Gustafsson JA, Dahlman-Wright K Association of estrogen receptor beta gene polymorphisms with bulimic disease in women. Mol Psychiatry 2004; 9:28-34.

15. Uhl GR. Molecular genetics of substance abuse vulnerability: re- markable recent convergence of genome scan results. Ann N Y Acad Sci2004; 1025:1-1.

16 Brown VM, Ossadtchi A, Khan AH, Cherry SR, Leahy RM, Smith $D J$. High-throughput imaging of brain gene expression. Genome Res 2002; 12:244-54.

17. Bloom FE Http://Www. neurome.com [viewed 2004].

18. Mirnics K, Middleton FA, Lewis DA, Levitt P. Analysis of complex brain disorders with gene expression microarrays: schizophrenia as a disease of the synapse. Trends Neurosci 2001; 24:479-86.

19. Harrison PJ, Weinberger DR. Schizophrenia genes, gene expression, and neuropathology: on the matter of their convergence. Mol Psychiatry2005; 10:40-68.

20. Iwamoto K, Kakiuchi C, Bundo M, Ikeda K, Kato T. Molecular characterization of bipolar disorder by comparing gene expression profiles of post-mortem brains of major mental disorders. Mol Psychiatry2004; 9: 406-416.

21. Smits KM, Smits LJ, Schouten JS, Stelma FF, Nelemans P, Prins $M H$. Influence of SERTPR and STin2 in the serotonin transporter gene on the effect of selective serotonin reuptake inhibitors in depression: a systematic review. Mol Psychiatr2004; 9:433-441.

22. Wong ML, O'Kirwan F, Hannestad JP, Irizarry KJ, Elashoff D, Licinio J. St John's wort and imipramine-induced gene expression profiles identify cellular functions relevant to antidepressant action and novel pharmacogenetic candidates for the phenotype of antidepressant treatment response. Mol Psychiatry 2004; 9:237-51.

23. Nichols CD, Sanders-Bush E. A single dose of lysergic acid diethylamide influences gene expression patterns within the mammalian brain. Neuropsychopharmacol 2002; 26:634-42.

24. Malhotra AK, Murphy GM, Kennedy JL. Pharmacogenetics of psychotropic drug response. Am J Psychiatr 2004; 161:780-96.

25. Bondy B, Zill P. Pharmacogenetics and psychopharmacology. Curr Opin Pharmacol 2004;4:72-78.

26. Enoch MA and Goldman D. The genetics of alcoholism and alcohol abuse. Curr Psychiatry Rep 2001; 3:144-51.

27. Cirelli C, Tononi G. Gene expression in the brain across the sleepwaking cycle. Brain Res 2000; 885:303-21.

28. Vawter MP, Evans S, Choudary P, Tomita H, Meador-Woodruff J, Molnar M, Li J, et al. Gender-specific gene expression in postmortem human brain: localization to sex chromosomes. Neuropsychopharmacol2004; 29:373-84.

29. Mong JA, Pfaff DW. Hormonal symphony: steroid orchestration of gene modules for sociosexual behaviors. Mol Psychiatry 2004; 9:550-6.

30. Payton A, Holland F, Diggle P, Rabbitt P, Horan M, Davidson $Y$, Gibbons $L$, et al. Cathepsin D exon 2 polymorphism associated with general intelligence in a healthy older population. Mol Psychiatr 2003; 8:14-8.

31. Silva AJ, Josselyn SA Cognitive neuroscience: The molecules of forgetfulness Nature 2002; 418, 929-930.

32. De Kloet ER. Hormones and the stressed brain. Ann N Y Acad Sc 2004; 1018:1-15.

33. Yalcin B, Willis-Owen SA, Fullerton J, Meesaq A, Deacon RM, Rawlins JN, et al. Genetic dissection of a behavioral quantitative trait locus shows that Rgs2 modulates anxiety in mice. Nature Genet 2004; 36:1197-202.

34. Bartlett CW, Flax JF, Logue MW, Vieland VJ, Bassett AS, Tallal P, Brzustowicz LM. A major susceptibility locus for specific language impairment is located on 13q21. Am J Hum Genet 2002; 71: 45-55. 